\title{
EFFECT OF DIFFERENT BONDING SUBSTRATES ON FRACTURE RESISTANCE OF THIN AND ULTRA-THIN OCCLUSAL VENEERS CONSTRUCTED USING DIFFERENT CAD/CAM MATERIALS
}

\author{
Shereen M. Elsayed*
}

\begin{abstract}
Objectives: The aim of this study was to compare the fracture resistance of thin and ultra-thin occlusal veneers constructed from glass ceramics and hybrid ceramics bonded to different bonding substrates after thermomechanical aging.

Materials and Methods: A total of 96 extracted maxillary first premolars were used. The teeth were divided into three equal groups according to the bonding substrate/protocol: EN, IDS Dentin, DDS Dentin. Each group was further subdivided into two equal subgroups according to the material of construction: E.max CAD, Vita Enamic. Each subgroup was further subdivided into two equal subdivisions according to the thickness of the occlusal veneer: Ultrathin, Thin. All the teeth were prepared to receive occlusal veneers. All the occlusal veneers were constructed using CAD/CAM technique. All occlusal veneers were cemented using adhesive resin cement. Thermomechanical aging was applied on all samples. Fracture resistance testing was performed.
\end{abstract}

Results: There was statistically significant difference between bonding protocols where IDS Dentin showed the statistically significantly highest mean fracture resistance followed by Enamel then DDS Dentin. Vita Enamic showed statistical significant higher mean fracture resistance than E.max CAD. Ultra-thin occlusal veneers showed statistical significant lower mean fracture resistance than thin occlusal veneers.

Conclusions: Ultrathin occlusal veneers must be cautiously used when glass ceramics is chosen. However, this minimal thickness could be a favorable option on using hybrid ceramics. Increasing the occlusal thickness to $0.5-0.8 \mathrm{~mm}$ could be promising upon bonding with either enamel or dentin with both materials used. Ultra-thin occlusal veneers must be used carefully upon bonding to dentin (IDS must be applied).

KEYWORDS: Thin occlusal veneers, Ultrathin occlusal veneers, Vita Enamic, E.max CAD, Glass ceramics, Hybrid ceramics, Thermomechanical aging.

\footnotetext{
* Associate Professor, Fixed Prosthodontics Department, Faculty of Dentistry, Cairo University, Egypt. Associate Professor,
} Fixed Prosthodontics Division, Oral and Maxillofacial Department, Faculty of Dentistry, Umm Al-Qura University. 


\section{INTRODUCTION}

Severely worn dentition has always been considered as a clinically challenging situation which is constantly overlooked until extensive prosthetic rehabilitation becomes mandatory. Tooth wear may result from several erosive, abrasive and attritive causes. ${ }^{(1)}$ Occlusal surfaces of premolars and molars may suffer from dental erosion while its progression goes on slowly and the diagnosis is obscure as well. ${ }^{(2,3)}$ The extent of tooth wear plays an important role in achieving the appropriate treatment decision. ${ }^{(4,5)}$

The extent of mutilation of the eroded worn dentition is irregular and may involve enamel only, enamel and minor part of dentin or enormous part of dentin with or without enamel margin. ${ }^{(6-8)}$ The occlusal destruction of the enamel and dentin will result in loss of occlusal contacts and in the worst-case scenarios, it may be compensated by overeruption rendering the situation with no place for the restorative material unless a substantial amount of tooth reduction took place. ${ }^{(9)}$

The demand for the minimally invasive concepts nowadays gives rise to the necessity for additive restorative options rather than the conventional subtractive options which needs additional removal of healthy sound hard tooth tissue for treatment of this complicated clinical conditions. ${ }^{(10)}$

The option of posterior occlusal veneers has been introduced based on the bonding principles applied in the anterior laminate veneer restorations. (11) Upon dealing with these restorations in which their durability and mechanical properties depends merely on adhesion between tooth, cement, and ceramic interfaces, the bonding substrate in conjunction with the type and the thickness of the ceramic materials must play a great role within the occlusal veneer restorative option. ${ }^{(12)}$ Thus, the type of the tooth tissue whether it is enamel, dentin or combined (dentin with peripheral enamel margin) will significantly affect the choice of the restorative material, its thickness, and consequently the bond strength values and the fracture strength of the whole complex. ${ }^{(13-15)}$ Superior adhesive bonding to enamel rather than dentin is well-known because both enamel and dentin are unique specialized tissues yet different in nature. ${ }^{(16)}$ The restorative material used for occlusal veneers will replace the missing tissue whether it is enamel or dentin, however the imitation of the dentino-enamel junction via an excellent durable restoration/tooth bond is mandatory to render the ultra-thin restoration a true successful composite complex. ${ }^{(16)}$

Adisagreement was revealed nowadays regarding the durability of the occlusal veneer bonding to enamel or dentin. Some authors emphasized superior bonding of lithium disilicate occlusal veneers when bonded to enamel even with reduced thickness. (17) Another study showed difference in fracture strength values between different thin occlusal veneer materials upon bonding to dentin with peripheral enamel and the authors related this difference to the inferior bonding to dentin. ${ }^{(18)}$ Ioannidis et al ${ }^{(1)}$ found no significant difference in load-bearing capacity between thin occlusal veneers of different materials when bonding substrate was limited to enamel.

Whenever discussing the minimally invasive restorations, it is always accompanied with efficient bonding strategies to assure reliable fracture resistance values. It was always underlined formerly that the percentage of enamel or dentin left could affect the success of the bonding mechanism and consequently the fracture resistance of ultrathin occlusal veneers. ${ }^{(15)}$ Many studies showed that bonding to dentin is still a limiting factor for durable adhesion and a dentin outline could lead to increased failure risk. ${ }^{(19-21)}$ Accordingly, the weakest link of the dentin-cement junction was improved by applying immediate dentin sealing concept (IDS). (12,22-25) This concept advocated the immediate treatment of the freshly cut dentin 
after its exposure by dentin bonding agent. ${ }^{(9)}$ IDS allows improvement of bond values, decrease microleakage and hypersensitivity, conservation of tooth structure, reduced gap formation and better restoration. ${ }^{(9,12,26)}$ IDS concept proved its efficacy in improvement of dentin bonding of anterior laminate veneers in a recent study. ${ }^{(27)}$ However, Van den Breemer ${ }^{(28)}$ found no significant difference between IDS and delayed dentin sealing (DDS) for posterior occlusal veneers.

The choice of the type of the restorative materials is one of the main key factors that will affect the success of posterior occlusal veneers. ${ }^{(1)}$ With the dental market being full of enormous restorative material options, the prosthodontist could decide the type and thickness of the restorative material which suits every clinical situation to achieve the best clinical outcomes. One of the most widely used all-ceramic systems is the lithium disilicate glass ceramics which combined the excellent mechanical strength with good esthetic properties, chemical stability, and biocompatibility. ${ }^{(29,30)}$

Another group of distinctive materials used nowadays successfully are the hybrid ceramics. A unique technique has been used within this group of materials, which is the combination between the polymer and ceramic materials to combine the benefits of both. ${ }^{(1)}$ The ceramic network $(86 \%)$ is infiltrated with a polymer material (14\%) to enhance the mechanical properties and the machinability of this material. ${ }^{(1,14,31)}$ This polymer-infiltrated ceramic network (PICN) material has been suggested as an alternative material to glass ceramics. ${ }^{(31)}$ The advantage of the elastic deformation properties of this material because of the inclusion of the polymeric part increases the load-bearing capacity of the restoration. (1) Additionally, it was assumed previously that polymer-infiltrated ceramics showed improved mechanical performance after luting of minimally invasive restorations with a reduced thickness owing to minimizing the crack propagation through the interlinked polymer network. ${ }^{(31)}$

A significant factor affecting the fracture strength of the occlusal veneers is the thickness of the restorative material. ${ }^{(8)}$ Even though the minimum acceptable thickness of ultra-thin occlusal veneers is not yet agreed upon in the literature, few studies have reported that lithium disilicate ceramics with 0.6$1 \mathrm{~mm}$ thickness can withstand occlusal forces up to 800 N. ${ }^{(7,31)}$ Another study revealed an acceptable fracture strength of pressable glass ceramic occlusal veneers with $0.5 \mathrm{~mm}$ thickness. ${ }^{(32)}$ On the contrary, a previous study concluded that alterations in ceramic occlusal veneers thickness influenced the fracture resistance significantly. They recommended that the thickness of the ceramic occlusal veneer must not be less than $0.7-1 \mathrm{~mm}$ regardless of the bonding substrate. ${ }^{(7)}$

Thus, the interest of the author has been raised to the question of whether the $0.3 \mathrm{~mm}$ minimal non-prep veneers that has been used effectively in the anterior region could be applied successfully in the posterior region or not. Can the interaction between the developed updated tools of the modern high-performance ceramics, hybrid ceramics, the CAD/CAM technology and the improved adhesive bonding concepts allow the application of thinner restorations while maintaining the high fracture resistance values or not. Therefore, the aim of this study was to compare the fracture resistance values after thermomechanical aging of thin and ultra-thin posterior occlusal veneers constructed from different ceramic and hybrid ceramic materials with different thicknesses bonded to different bonding substrates. The null hypotheses were: 1 . There would be no effect of different bonding substrates and protocols on fracture resistance values of posterior occlusal veneers. 2. There would be no effect of different materials on fracture resistance values of posterior occlusal veneers. 3 . There would be no effect of different occlusal veneers thicknesses on fracture resistance values. 


\section{MATERIALS AND METHODS}

\section{Teeth selection:}

A total of 96 recently extracted non-carious, non-restored unworn maxillary first premolars with similar dimensions bucco-lingually and mesiodistally were collected and cleaned with ultrasonic scalers. The chosen teeth were inspected for absence of cracks using transmitted light, those with cracks were discarded and replaced by another crack-free ones. Teeth were stored in saline solution at room temperature until the beginning of the experiment.

The roots of the teeth were covered with $0.25 \mathrm{~mm}$ thickness of heated modelling wax $2 \mathrm{~mm}$ below the CEJ to simulate the periodontal membrane. The teeth were placed in a polyvinylchloride tubes filled with soft epoxy resin (Chemapoxy 150, CMB, Giza, Egypt) $2 \mathrm{~mm}$ apical to the CEJ and were left until complete setting.

\section{Sample Grouping:}

All the selected teeth were randomly divided into three equal groups ( $n=32)$ according to the bonding substrate and bonding protocol as follows:

Group 1 (EN group): The selected teeth were prepared in the enamel without invading the dentin to simulate attritive defect in the occlusal surface.

Group 2 (IDS Dentin): The selected teeth were prepared in the dentin with enamel margin all around + application of Immediate Dentin Sealing protocol.

Group 3 (DDS Dentin): The selected teeth were prepared in the dentin with enamel margin all around + application of Conventional Delayed Dentin Sealing protocol.

Each group was further subdivided into two equal subgroups ( $n=16)$ according to the material of construction of the occlusal veneers as follows:

Subgroup I (E.max CAD): Occlusal veneers were constructed from CAD/CAM lithium disilicate glass ceramics.

Subgroup II (Vita Enamic): Occlusal veneers were constructed from $\mathrm{CAD} / \mathrm{CAM}$ hybrid-ceramics.

Each subgroup was further subdivided into two equal subdivisions $(n=8)$ according to the thickness of the occlusal veneer as follows:

Subdivision A (Ultra-thin): Occlusal veneers were constructed with $0.3 \mathrm{~mm}$ thickness at the fissure and $0.6 \mathrm{~mm}$ thickness at the cusps.

Subdivision B (Thin): Occlusal veneers were constructed with $0.5 \mathrm{~mm}$ thickness at the fissure and $0.8 \mathrm{~mm}$ thickness at the cusps.

Flow chart showing sample grouping is presented in Fig. (1).

\section{Natural teeth}

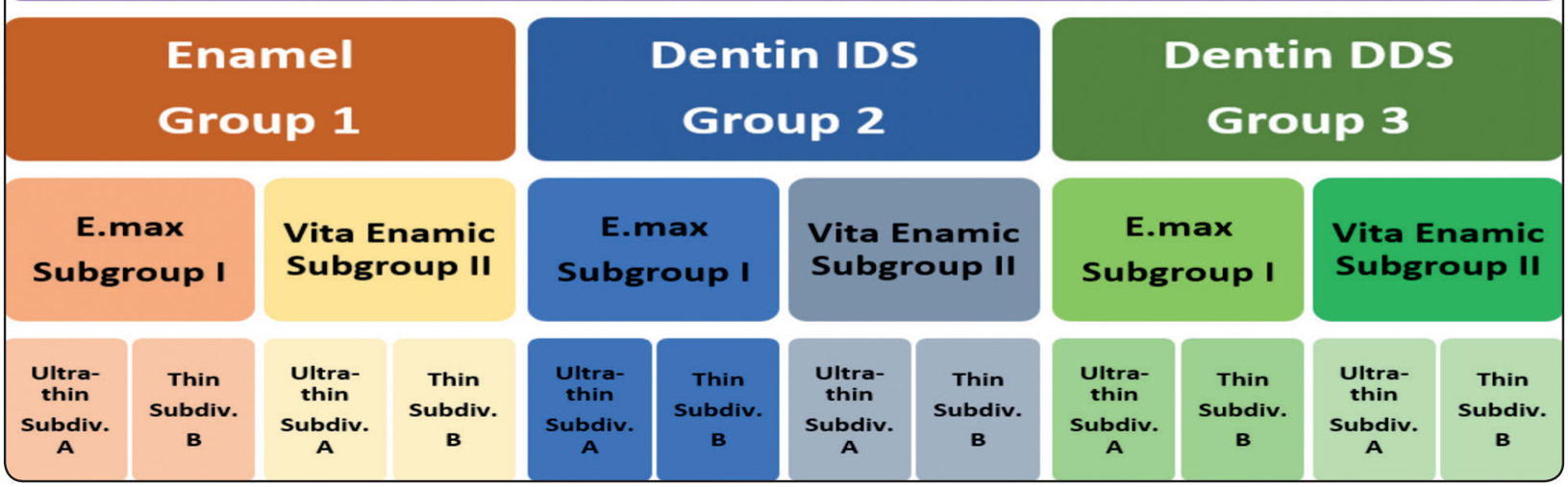

Fig. (1): Flow chart showing sample grouping. 


\section{Teeth Preparation:}

All the teeth were prepared to receive occlusal veneers with enamel margins for all groups, subgroups, and subdivisions. Regarding the EN group, the selected teeth were prepared in the enamel without invading the dentin. The preparation of the teeth was done using a handpiece that is connected to custom-made paralleling machine and an angulated adaptor with $120^{\circ}$. The enamel layer of the occlusal surface was reduced by $0.3 \mathrm{~mm}$ depth of reduction at the fissures and $0.5 \mathrm{~mm}$ depth of reduction at the cusps with $120^{\circ}$ angles between buccal and lingual cusps, mesiobuccal and distobuccal cusp slopes, and mesiolingual and distolingual cusp slopes. Roundation of all sharp angles was done. Smoothness of the whole preparation was done using rubber polishing kit. ${ }^{(6,8,14)}$

As for the IDS Dentin and DDS Dentin groups, teeth preparation was done using the same technique and paralleling device as the enamel group, but reduction of the occlusal surface was extended into the dentin with $0.5 \mathrm{~mm}$ depth of reduction at the fissures and $0.8 \mathrm{~mm}$ depth of reduction at the cusps. ${ }^{(8)}$ Fig. (2).

As regards to group 2 (IDS Dentin group), the immediate dentin sealing protocol (IDS) was utilized directly after preparation on the freshly prepared dentin surface. ${ }^{(12,33)}$ Etching of the dentin surface was done using $37 \%$ phosphoric acid etchant (N-Etch Etching gel, Ivoclar Vivadent) for $15 \mathrm{sec}$. then rinsed with water for $20 \mathrm{sec}$. followed by $5 \mathrm{sec}$. gentle-air dryness. A thin layer of adhesive resin (Tetric N-bond Adhesive, Ivoclar Vivadent) was applied subsequently on the dentin gently by brush without air-thinning, followed by light-curing for $20 \mathrm{sec}$. using light curing unit at $1000 \mathrm{~mW} / \mathrm{cm}^{2}$ (Bluephase N, Ivoclar Vivadent). Finally, a thin layer of flowable composite resin (Tetric N-Flow, Ivoclar Vivadent) was applied on the dentin surface only and light-cured for $40 \mathrm{sec}$. Excess resin was removed from the all-around enamel margin meticulously using low-speed rubber points (8856 P 314 018, Komet, 9608314 030, Komet).

Concerning group 3 (DDS Dentin group), the conventional delayed dentin sealing (DDS) protocol was applied after preparation by two weeks at the time of cementation of the occlusal veneers.

\section{Occlusal Veneers Fabrication:}

All the prepared teeth were digitally scanned using InEos X5 scanner (Dentsply Sirona, Germany). All the scanned exposures were sent to the CAD/CAM software (exocad DentalCAD, exocad $\mathrm{GmbH}$, Germany) to design the occlusal veneers. Occlusal veneers were designed with a

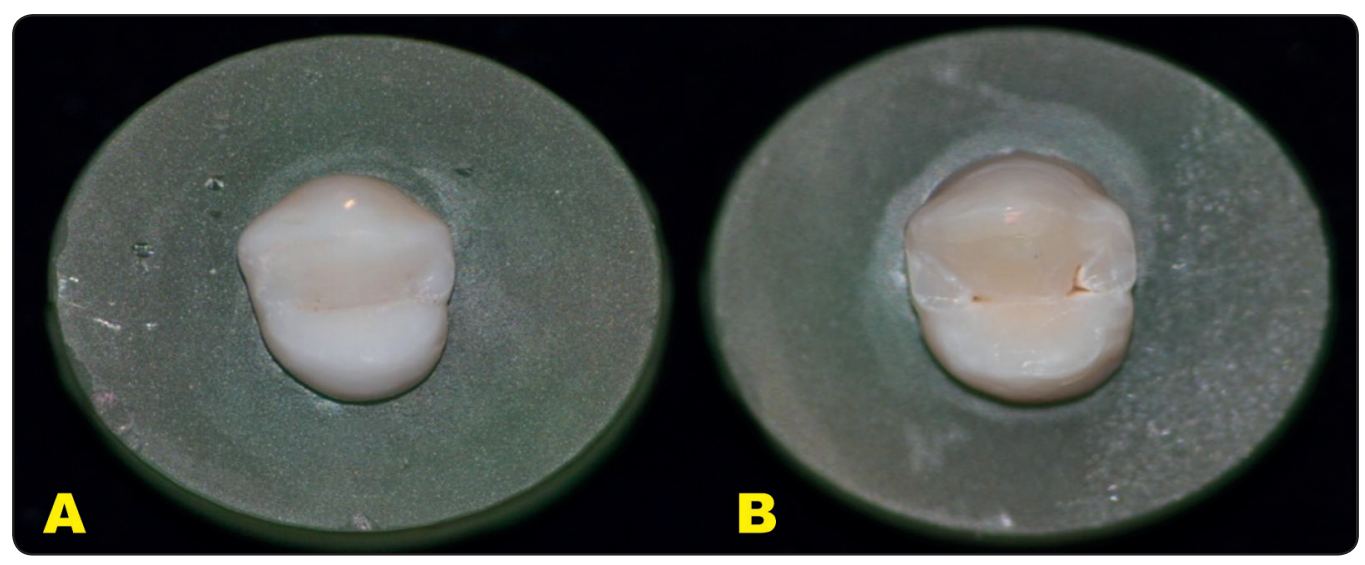

Fig. (2): Preparation design for A) Enamel group, B) Dentin group. 
semi-anatomic shape to ensure standardization of ceramic thickness within the samples of each group, subgroup, and subdivision. ${ }^{(8)}$ Cement space was adjusted to be $30 \mu \mathrm{m}$ for all occlusal veneers to allow passive fit. ${ }^{(34)}$

Regarding the EN group (group 1), subgroup I subdivision A and subgroup II subdivision A, the thickness of the virtual design of the occlusal veneers was adjusted to be $0.3 \mathrm{~mm}$ in the central groove, $0.6 \mathrm{~mm}$ at the cusps and $0.5 \mathrm{~mm}$ at the internal cusp slopes. The same parameters were applied for the dentin groups 2 and 3, subgroups I and II subdivision A.

As for the EN group (group 1), subgroup I subdivision B and subgroup II subdivision B, the thickness of the virtual design of the occlusal veneers was adjusted to be $0.5 \mathrm{~mm}$ in the central groove, $0.8 \mathrm{~mm}$ at the cusps and $0.7 \mathrm{~mm}$ at the internal cusp slopes. The same parameters were applied for the dentin groups 2 and 3, subgroups I and II subdivision B. Virtual designing was done by the same operator.

After finalizing the occlusal veneer design parameters for all the assigned groups, IPS E.max CAD block (IPS e.max CAD, Ivoclar Vivadent, Shaan Liechtenstein) and Vita Enamic block (Vita Enamic, Vita Zahnfabrik) were selected to start the milling process. Milling of the occlusal veneers was done using CAD/CAM milling machine (Ceramill Motion 2,Ammann Girrbach,Austria).All the milled occlusal veneers for all groups were examined for seating and fitting on their respective teeth in each group, subgroup, and subdivision using magnifying lens. Afterwards, IPS E.max CAD occlusal veneers were crystallized in a special furnace (Programat EP 5000, Ivoclar Vivadent) according to manufacturer instructions. Vita Enamic occlusal veneers were finished and polished only using different polishing instruments (Vita Enamic Polishing Set, Vita Zahnfabrik) without any postmilling sintering according to manufacturer instructions. Fig. (3).

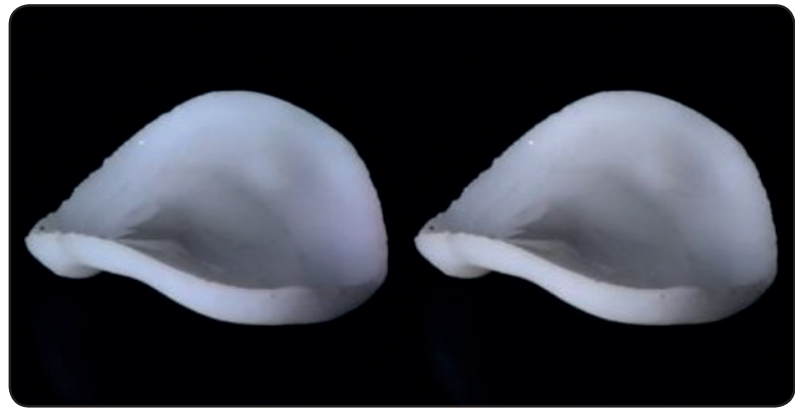

Fig. (3): Occlusal veneers after milling and finishing.

\section{Adhesive cementation of the occlusal veneers:}

\section{Surface treatment of the fitting surface of the IPS E.max CAD occlusal veneers:}

The fitting surfaces of IPS E.max CAD occlusal veneers were treated with 5\% hydrofluoric acid etchant (IPS ceramic etchant gel, Ivoclar Vivadent) for $20 \mathrm{sec}$. followed by $60 \mathrm{sec}$. water rinsing then dryness. A silane coupling agent (Monobond plus, Ivoclar Vivadent) was applied on the fitting surface for $60 \mathrm{sec}$

\section{Surface treatment of the fitting surface of Vita En- amic occlusal veneers:}

The fitting surfaces of the Vita Enamic occlusal veneers were treated with $5 \%$ hydrofluoric acid etchant for $60 \mathrm{sec}$. according to manufacturer instructions, followed by $60 \mathrm{sec}$. rinsing with water spray then dryness. A silane coupling agent was placed for $60 \mathrm{sec}$.

\section{Surface treatment of natural teeth:}

For adhesive cementation, all the teeth used in this study were assigned for total-etch adhesive technique. Regarding the EN group, the enamel surface was etched using 37\% phosphoric acid ge for $30 \mathrm{sec}$., rinsed with copious water for $15 \mathrm{sec}$. then air-dried. Adhesive resin (Tetric N-bond Adhesive, Ivoclar Vivadent) was applied to the etched enamel and allow to react for $15 \mathrm{sec}$. then air-dried for $5 \mathrm{sec}$. without polymerization. 
As for group 2 with IDS Dentin protocol, the IDS layer was treated with a chair-side air-abrasion intra-oral sandblaster (Cojet, 3M ESPE) with 40 $\mu \mathrm{m} \mathrm{Al}_{2} \mathrm{O}_{3}$ particles and 30 PSI until the surface becomes matt. The enamel margin was etched using $37 \%$ phosphoric acid for $30 \mathrm{sec}$. then rinsed. Silane coupling agent was applied to the IDS layer and left to react for $60 \mathrm{sec}$. then adhesive resin was applied to the whole prepared surface for $20 \mathrm{sec}$. followed by air-dryness without polymerization. ${ }^{(12,16)}$

Concerning group 3 with DDS Dentin protocol, enamel margin was etched with $37 \%$ phosphoric acid for $30 \mathrm{sec}$. and dentin surface for $10 \mathrm{sec}$. followed by copious rinsing with water. Adhesive resin was applied to the whole prepared surface for $20 \mathrm{sec}$. followed by air-dryness without polymerization. ${ }^{(12)}$

All occlusal veneers were cemented to their respective teeth using adhesive resin cement (Variolink Esthetic adhesive resin cement, Ivoclar Vivadent). Fig (4). The cement was applied to the fitting surfaces of all the occlusal veneers which were placed gently on their respective teeth and held under finger pressure for five minutes. The excess cement was removed from all sides using cotton pellets then light polymerization was done using light curing unit for $40 \mathrm{sec}$. from each side.

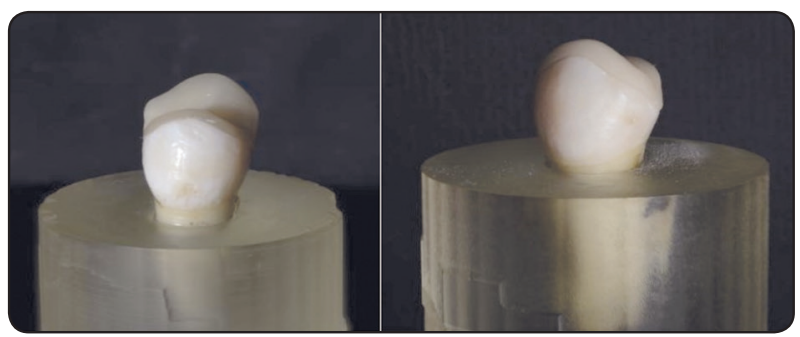

Fig. (4): Occlusal veneer cemented to its respective tooth.

\section{Thermocycling}

Laboratory simulations of clinical service as thermocycling and mechanical aging are often performed to simulate clinical intra-oral conditions. The number of thermal cycles used in this study was 1200 cycles corresponding to 6 months. Dwell times were $25 \mathrm{sec}$. in each water bath with an interval time of $10 \mathrm{sec}$. The low-temperature point was $5{ }^{\circ} \mathrm{C}$. The high temperature point was $55^{\circ} \mathrm{C}$.

\section{Mechanical Aging:}

The mechanical aging test was performed using the ROBOTA mastication simulator integrated with a thermocyclic protocol and operated by a servo motor (model ACH -09075DC-T, AD - Tech Technology CO., LTD., Germany). ROBOTA chewing simulator has four chambers that simultaneously simulate the vertical and horizontal movements in the thermodynamic state. Each of the chambers consists of a hardened steel upper pin holder that can be tightened with a screw to serve as an antagonist, and a plastic lower specimen holder in which the specimen can be embedded. Occlusal veneers cemented on the respective teeth and embedded in plastic moulds were fixed in the lower specimen holder. A weight of $5 \mathrm{~kg}$ was exerted, corresponding to a masticatory force of $49 \mathrm{~N}$. The test was duplicated 75000 times to clinically mimic 6 months of chewing load. Fig. (5).

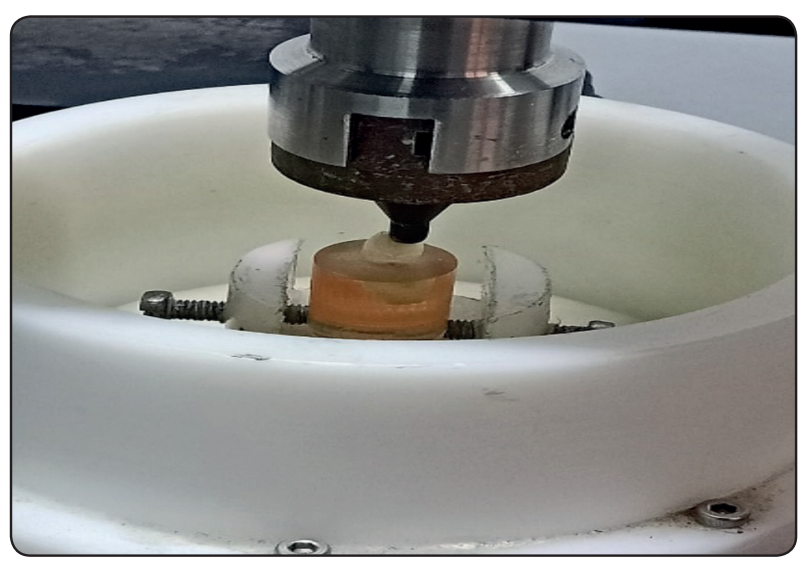

Fig. (5): Mechanical Aging Testing.

\section{Fracture Resistance Testing}

All specimens were mounted individually on a computer-controlled materials testing machine (model 3345; Instron Industrial Products, Norwood, MA, USA) with a load cell of $5 \mathrm{kN}$, and data were recorded using computer software (Instron ${ }^{\circledR}$ 
Bluehill Lite Software). Specimens were secured in the lower fixed compartment of the testing machine by tightening bolts. Fracture testing was performed with a compressive force applied occlusally with a metal rod and a spherical tip (3.8 $\mathrm{mm}$ diameter) attached to the upper moving chamber of the testing machine and moving at a crosshead speed of $1 \mathrm{~mm} / \mathrm{min}$, with a tin foil in between to achieve a homogeneous stress distribution and minimize the transmission of local force peaks. The failure load manifested itself by an audible crack and was confirmed by a sharp drop in the load-deflection curve recorded by computer software (Bluehill Lite Software Instron ${ }^{\circledR}$ Instruments). The load required to fracture was recorded in Newton. Fig. (6).

\section{Statistical Analysis}

Numerical data were explored for normality by checking the distribution of data and using tests of normality (Kolmogorov-Smirnov and Shapiro-

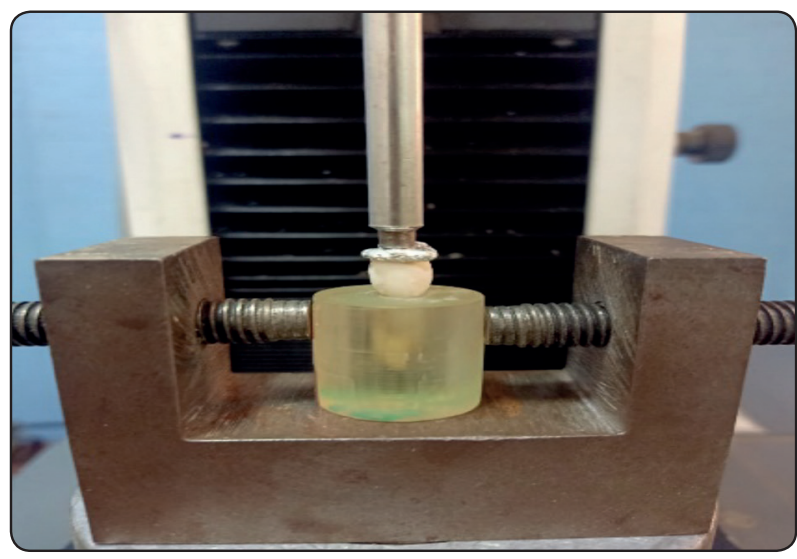

Fig. (6): Fracture resistance Testing.
Wilk tests). All data showed normal (parametric) distribution. Data were presented as mean and standard deviation (SD) values. Three-way Analysis of Variance (ANOVA) was used to study the effect of bonding protocol, ceramic type, thickness and their interactions on mean fracture resistance. Bonferroni's post-hoc test was used for pair-wise comparisons when ANOVA test is significant. The significance level was set at $\mathrm{P} \leq 0.05$. Statistical analysis was performed with IBM SPSS Statistics for Windows, Version 23.0. Armonk, NY:IBM Corp.

\section{RESULTS}

\section{Three-way ANOVA results}

The results showed that bonding protocol (regardless of ceramic type and thickness) had a statistically significant effect on mean fracture resistance $(P$-value $<0.001$, Effect size $=0.858)$. Ceramic type (regardless of bonding protocol and thickness) had a statistical significant effect on mean fracture resistance $(P$-value $<0.001$, Effect size $=0.475)$. Thickness (regardless of ceramic type and bonding protocol) had a statistical significant effect on mean fracture resistance $(P$-value $<0.001$, Effect size $=0.842)$. The interaction between the three variables had statistically significant effect on mean fracture resistance $(P$-value $=0.001$, Effect size $=0.151)$. Since the interaction between the variables is statistically significant, so the variables are dependent upon each other. Table (1).

TABLE (1) Three-way ANOVA results for the effect of different variables on mean fracture resistance (N).

\begin{tabular}{lcccccc}
\hline & $\begin{array}{c}\text { Type III Sum of } \\
\text { Squares }\end{array}$ & df & Mean Square & $F$-value & $P$-value & $\begin{array}{c}\text { Effect size } \\
\text { (Partial eta } \\
\text { Squared) }\end{array}$ \\
\hline Source of variation & 351404.6 & 1 & 351404.6 & 76.058 & $<0.001^{*}$ & 0.475 \\
Beramic type & 2347806.9 & 2 & 1173903.5 & 254.078 & $<0.001^{*}$ & 0.858 \\
Thickness & 2074737.4 & 1 & 2074737.4 & 449.053 & $<0.001^{*}$ & 0.842 \\
Ceramic type 'Bonding protocol' & 68905.5 & 2 & 34452.8 & 7.457 & $0.001^{*}$ & 0.151 \\
Thickness interaction & & & &
\end{tabular}

$d f:$ degrees of freedom $=(n-1), *$ : Significant at $P \leq 0.05$ 


\section{Effect of bonding protocol regardless of ce- ramic type and thickness}

Regardless of ceramic type and thickness, there was a statistically significant difference between bonding protocols $(P$-value $<0.001$, Effect size $=$ 0.858). Pair-wise comparisons between protocols revealed that IDS Dentin showed the statistically significantly highest mean fracture resistance. Enamel showed statistically significantly lower mean value. DDS Dentin showed the statistically significantly lowest mean fracture resistance. Table (2).

\section{Effect of ceramic type regardless of bonding protocol and thickness}

Regardless of bonding protocol and thickness, Vita Enamic revealed statistical significant higher fracture resistance values than E.max CAD ( $P$-value $<0.001$, Effect size $=0.475)$. Table (3).

\section{Effect of thickness regardless of ceramic type and bonding protocol}

Regardless of ceramic type and bonding protocol, $0.3-0.6 \mathrm{~mm}$ thickness showed statistical significant lower mean fracture resistance values than $0.5-0.8 \mathrm{~mm}$ thickness $(P$-value $<0.001$, Effect size $=0.842)$. Table $(4)$.

TABLE (2) The mean, standard deviation (SD) values and results of three-way ANOVA test for comparison between fracture resistance $(\mathrm{N})$ of bonding protocols regardless of ceramic type and thickness.

\begin{tabular}{|c|c|c|c|c|c|c|c|}
\hline \multicolumn{2}{|c|}{ Enamel } & \multicolumn{2}{c|}{ DDS Dentin } & \multicolumn{2}{c|}{ IDS Dentin } & \multirow{2}{*}{ P-value } & $\begin{array}{c}\text { Effect size (Partial eta } \\
\text { squared) }\end{array}$ \\
\cline { 1 - 4 } Mean & SD & Mean & SD & Mean & SD & & 0.858 \\
\hline
\end{tabular}

*: Significant at $P \leq 0.05$, Different superscripts indicate statistically significant difference

TABLE (3) The mean, standard deviation (SD) values and results of three-way ANOVA test for comparison between fracture resistance $(\mathrm{N})$ of the two ceramic types regardless of bonding protocol and thickness.

\begin{tabular}{|c|c|c|c|c|c|}
\hline \multicolumn{2}{|c|}{ Vita Enamic } & \multicolumn{2}{c|}{ E.max CAD } & \multirow{2}{*}{ P-value } & $\begin{array}{c}\text { Effect size (Partial eta } \\
\text { squared })\end{array}$ \\
\cline { 1 - 3 } Mean & SD & Mean & SD & & $<0.001 *$ \\
\hline 927.1 & 226.7 & 806.1 & 303 & 0.475 \\
\hline
\end{tabular}

*: Significant at $P \leq 0.05$

Table (4). The mean, standard deviation (SD) values and results of three-way ANOVA test for comparison between fracture resistance $(\mathrm{N})$ of the two thicknesses regardless of ceramic types and bonding protocol.

\begin{tabular}{|c|c|c|c|c|c|}
\hline \multicolumn{2}{|c|}{$0.3-0.6 \mathrm{~mm}$} & \multicolumn{2}{c|}{$0.5-0.8 \mathrm{~mm}$} & \multirow{2}{*}{$P$-value } & $\begin{array}{c}\text { Effect size (Partial } \\
\text { eta squared) }\end{array}$ \\
\cline { 1 - 3 } Mean & SD & Mean & SD & $<0.001^{*}$ & 0.842 \\
\hline
\end{tabular}

\footnotetext{
*: Significant at $P \leq 0.05$
} 
Effect of different interactions on fracture resistance:

a) Comparison between bonding protocols with different interactions of variables (Table 5. Fig.7):

Whether with Vita Enamic or E.max CAD at thickness $0.3-0.6 \mathrm{~mm}$, there was a statistically significant difference between bonding protocols. Pair-wise comparisons between protocols revealed that IDS Dentin showed the statistically significantly highest mean fracture resistance. There was no statistically significant difference between Enamel and DDS Dentin protocols; both showed the statistically significantly lowest mean fracture resistance values.

While with Vita Enamic or E.max CAD at thickness $0.5-0.8 \mathrm{~mm}$, there was a statistically significant difference between bonding protocols. Pair-wise comparisons between protocols revealed that Enamel bonding protocol showed the statistically significantly highest mean fracture resistance. IDS Dentin bonding protocol showed statistically significantly lower mean value. DDS Dentin bonding protocol showed the statistically significantly lowest mean fracture resistance.

TABLE (5).The mean, standard deviation (SD) values and results of three-way ANOVA test for comparison between fracture resistance $(\mathrm{N})$ of different bonding protocols with different interactions of variables.

\begin{tabular}{|l|l|c|c|c|c|c|c|c|c|}
\hline \multirow{2}{*}{ Ceramic } & \multirow{2}{*}{ Thickness } & \multicolumn{2}{|c|}{ Enamel } & \multicolumn{2}{c|}{ DDS Dentin } & \multicolumn{2}{c|}{ IDS Dentin } & \multirow{2}{*}{ P-value } & $\begin{array}{c}\text { Effect size (Partial } \\
\text { eta squared) }\end{array}$ \\
\cline { 3 - 10 } & & Mean & SD & Mean & SD & Mean & SD & & 0.345 \\
\hline \multirow{2}{*}{$\begin{array}{l}\text { Vita } \\
\text { Enamic }\end{array}$} & $0.3-0.6 \mathrm{~mm}$ & $742.5^{\mathrm{B}}$ & 41.2 & $727.4^{\mathrm{B}}$ & 35.7 & $930.4^{\mathrm{A}}$ & 80.9 & $<0.001^{*}$ & 0.764 \\
\cline { 2 - 11 } & $0.5-0.8 \mathrm{~mm}$ & $1328^{\mathrm{A}}$ & 70.1 & $768.4^{\mathrm{C}}$ & 70.5 & $1065^{\mathrm{B}}$ & 79.3 & $<0.001^{*}$ & 0.779 \\
\hline $\begin{array}{l}\text { E.max } \\
\text { CAD }\end{array}$ & $0.3-0.6 \mathrm{~mm}$ & $497.7^{\mathrm{B}}$ & 22.5 & $444.7^{\mathrm{B}}$ & 29.6 & $974.9^{\mathrm{A}}$ & 109.7 & $<0.001^{*}$ & 0.784 \\
\cline { 2 - 11 } & $0.5-0.8 \mathrm{~mm}$ & $1231^{\mathrm{A}}$ & 73.8 & $649.5^{\mathrm{C}}$ & 69 & $1038^{\mathrm{B}}$ & 78.3 & $<0.001^{*}$ & 0.3 \\
\hline
\end{tabular}

*: Significant at $P \leq 0.05$, Different superscripts in the same row indicate statistically significant difference between bonding protocols

Table (6). The mean, standard deviation (SD) values and results of three-way ANOVA test for comparison between fracture resistance $(\mathrm{N})$ of ceramic types with different interactions of variables.

\begin{tabular}{|c|c|c|c|c|c|c|c|}
\hline \multirow{2}{*}{$\begin{array}{l}\text { Bonding } \\
\text { protocol }\end{array}$} & \multirow{2}{*}{ Thickness } & \multicolumn{2}{|c|}{ Vita Enamic } & \multicolumn{2}{|c|}{ E.max CAD } & \multirow{2}{*}{$P$-value } & \multirow{2}{*}{$\begin{array}{c}\text { Effect size } \\
\text { (Partial eta } \\
\text { squared) }\end{array}$} \\
\hline & & Mean & SD & Mean & SD & & \\
\hline \multirow{2}{*}{ Enamel } & $0.3-0.6 \mathrm{~mm}$ & 742.5 & 41.2 & 497.7 & 22.5 & $<0.001 *$ & 0.382 \\
\hline & $0.5-0.8 \mathrm{~mm}$ & 1328.5 & 70.1 & 1231.2 & 73.8 & $0.005^{*}$ & 0.089 \\
\hline \multirow{2}{*}{ DDS Dentin } & $0.3-0.6 \mathrm{~mm}$ & 727.4 & 35.7 & 444.7 & 29.6 & $<0.001 *$ & 0.452 \\
\hline & $0.5-0.8 \mathrm{~mm}$ & 768.4 & 70.5 & 649.5 & 69 & $0.001 *$ & 0.127 \\
\hline \multirow{2}{*}{ IDS Dentin } & $0.3-0.6 \mathrm{~mm}$ & 930.4 & 80.9 & 974.9 & 109.7 & 0.194 & 0.02 \\
\hline & $0.5-0.8 \mathrm{~mm}$ & 1065.4 & 79.3 & 1038.6 & 78.3 & 0.433 & 0.007 \\
\hline
\end{tabular}

$*$ : Significant at $P \leq 0.05$ 


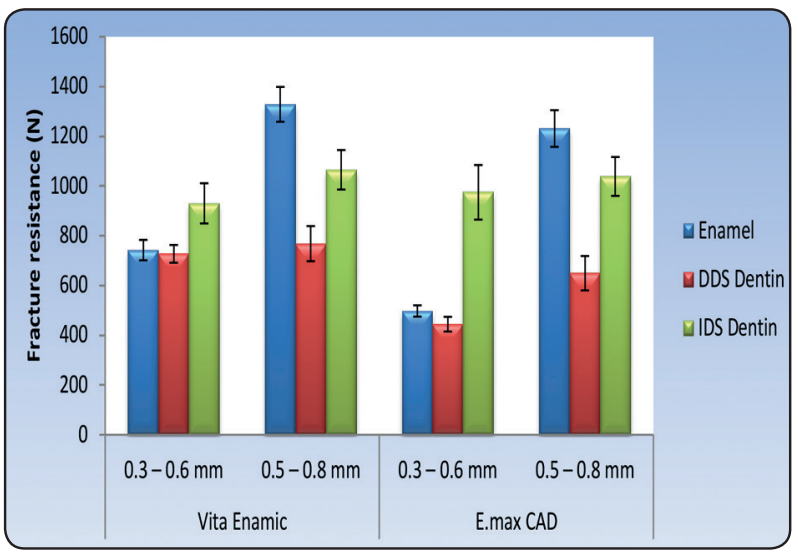

Fig. (7). Bar chart representing mean and standard deviation values for fracture resistance of bonding protocols.

\section{b) Comparison between ceramic types with differ- ent interactions of variables (Table 6, Fig.8):}

Whether with enamel or DDS Dentin bonding protocols using $0.3-0.6 \mathrm{~mm}$ or $0.5-0.8 \mathrm{~mm}$ thicknesses, Vita Enamic showed statistical significant higher mean fracture resistance than E.max CAD.

While with IDS Dentin bonding protocol using $0.3-0.6 \mathrm{~mm}$ or $0.5-0.8 \mathrm{~mm}$ thicknesses, there was no statistically significant difference between mean fracture resistance values of the two ceramic types.

\section{c) Comparison between thicknesses with different interactions of variables (Table 7, Fig.9):}

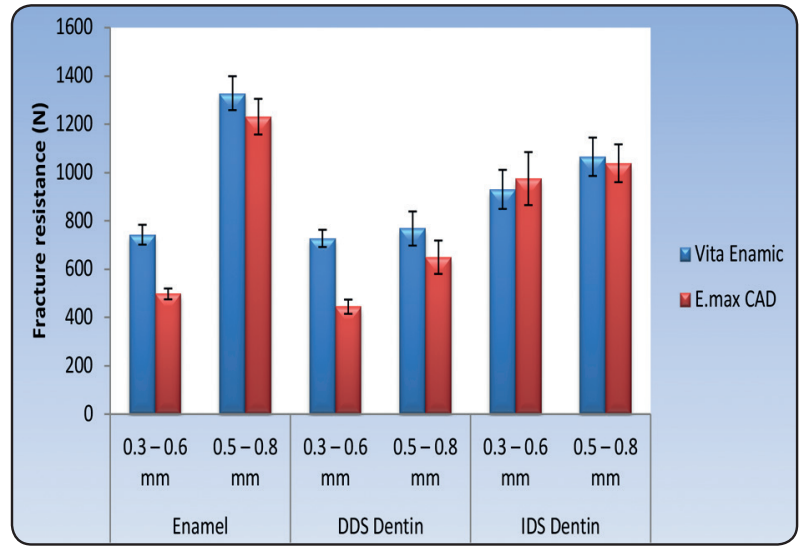

Fig. (8). Bar chart representing mean and standard deviation values for fracture resistance of the two ceramic types.

With Vita Enamic using Enamel or IDS Dentin bonding protocols, $0.3-0.6 \mathrm{~mm}$ thickness showed statistical significant lower fracture resistance values than $0.5-0.8 \mathrm{~mm}$ thickness. While with DDS Dentin bonding protocol; there was no statistically significant difference between the two thicknesses.

With E.max CAD using Enamel or DDS Dentin bonding protocols, $0.3-0.6 \mathrm{~mm}$ thickness showed statistical significant lower mean fracture resistance than $0.5-0.8 \mathrm{~mm}$ thickness. While with IDS Dentin bonding protocol; there was no statistically significant difference between the two thicknesses.

TABLE (7) The mean, standard deviation (SD) values and results of three-way ANOVA test for comparison between fracture resistance $(\mathrm{N})$ of the two thicknesses with different interactions of variables.

\begin{tabular}{|c|c|c|c|c|c|c|c|}
\hline \multirow{2}{*}{ Ceramic } & \multirow{2}{*}{ Bonding protocol } & \multicolumn{2}{|c|}{$0.3-0.6 \mathrm{~mm}$} & \multicolumn{2}{|c|}{$0.5-0.8 \mathrm{~mm}$} & \multirow{2}{*}{$P$-value } & \multirow{2}{*}{$\begin{array}{c}\text { Effect size } \\
\text { (Partial etc } \\
\text { squared) }\end{array}$} \\
\hline & & Mean & SD & Mean & SD & & \\
\hline \multirow{3}{*}{$\begin{array}{l}\text { Vita } \\
\text { Enamic }\end{array}$} & Enamel & 742.5 & 41.2 & 1328.5 & 70.1 & $<0.001 *$ & 0.78 \\
\hline & DDS Dentin & 727.4 & 35.7 & 768.4 & 70.5 & 0.231 & 0.017 \\
\hline & IDS Dentin & 930.4 & 80.9 & 1065.4 & 79.3 & $<0.001 *$ & 0.158 \\
\hline \multirow{3}{*}{$\begin{array}{l}\text { E.max } \\
\text { CAD }\end{array}$} & Enamel & 497.7 & 22.5 & 1231.2 & 73.8 & $<0.001 *$ & 0.847 \\
\hline & DDS Dentin & 444.7 & 29.6 & 649.5 & 69 & $<0.001 *$ & 0.302 \\
\hline & IDS Dentin & 974.9 & 109.7 & 1038.6 & 78.3 & 0.064 & 0.04 \\
\hline
\end{tabular}

*: Significant at $P \leq 0.05$ 


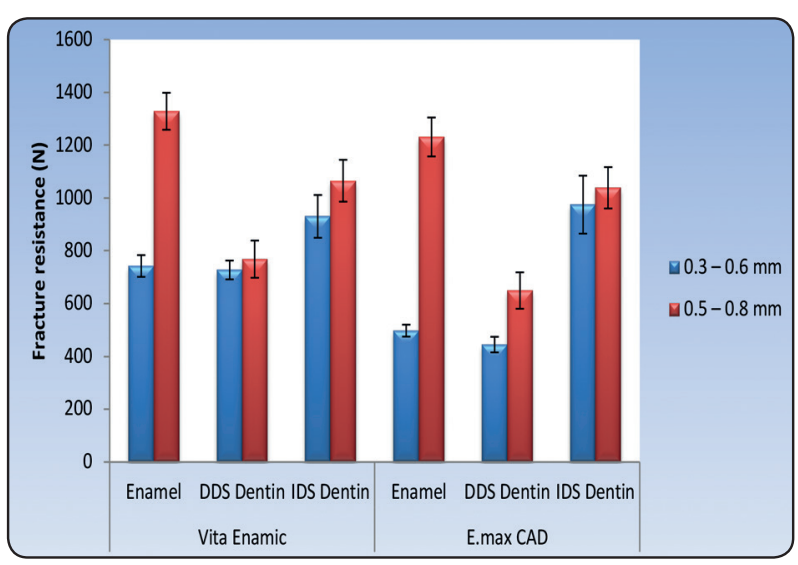

Fig. (9). Bar chart representing mean and standard deviation values for fracture resistance of the two thickness.

\section{DISCUSSION}

The term posterior occlusal veneer has gained wide acceptance nowadays in the dental field in an attempt to shift towards the minimally invasive alternatives after recognizable improvements in restorative materials, techniques, and adhesive systems. (31) The endurance of these innovative restorations is affected by multiple factors including restoration type and thickness, mechanical properties of restorative materials, the position of the tooth being restored, occlusal forces of the patient as well as the bonding substrate and bonding technique. ${ }^{(9,35,36)}$

All the teeth used in this study were prepared according to the preparation guidelines of minimally invasive restorations as stated in several previous studies. ${ }^{(14,37-40)}$ For standardization, all teeth were prepared using paralleling machine. CAD/CAM technique was advocated in this study for construction of all occlusal veneers to assure the standardization of all the design parameters of the occlusal veneers including restorative material thickness, the anatomy of the occlusal veneer, the internal fit of the occlusal veneer, and the mechanical strength of the material itself.

Total etch technique has been applied for cementation of all occlusal veneers in the current study due to the proven superior bonding of the etching step of the enamel separately upon comparing it with self-etch alternatives where they reveal lower etching ability to enamel. ${ }^{(41-43)}$ High fracture resistance was achieved when minimally invasive restorations were bonded using total-etch technique. ${ }^{(14,32,45)}$ Total etch technique revealed excellent bond strength values to enamel, but less values were reached when dentin is involved. ${ }^{(8,44)}$ Another study resulted in insufficient bond strength values between thin occlusal veneers and enamel due to the application of self-etching primer. ${ }^{(46)}$

Thermocycling was applied in this study with a range between $6.6^{\circ} \mathrm{C}$ and $55.5^{\circ} \mathrm{C}$ for 1200 cycle to simulate the temperature fluctuations normally found in the oral cavity equivalent to 6 months clinical service which significantly affects the bond strength values and consequently the fracture resistance values. ${ }^{(47)}$ Following thermal cycling, mechanical aging was performed using chewing simulator with 75000 cycles which represents 6 months chewing simulation. ${ }^{(16,48)}$ Fatigue fracture is the most common cause for most of the mechanical failures of ceramic restorations. Therefore, thermomechanical aging is considered mandatory to be able to relate the in-vitro study results to the performance of the restorative option under investigation so that the clinical guidelines could be suggested. ${ }^{(49-51)}$

The main goal of this study was to assess the influence of the critical reduction in thickness of different types of occlusal veneers beyond the manufacturer recommendations and its relationship with the bonding substrate and bonding protocols. It was assumed previously that bonding of thin ceramic occlusal veneers to enamel showed equivalent results as that of thick dentin-bonded occlusal veneers. ${ }^{(32,45)}$

Nevertheless, the null hypotheses stating that the different bonding substrates and protocols, different materials, as well as different occlusal veneers 
thicknesses would not affect the fracture resistance of occlusal veneers were all rejected.

The results of this study showed statistically significant difference between bonding substrates/ protocols regardless of the material and thickness of the occlusal veneers where IDS Dentin showing the highest mean fracture resistance values followed by En Group, while the DDS Dentin showed the least mean values $(1002.3 \mathrm{~N}, 950 \mathrm{~N}$ and $647.5 \mathrm{~N}$ respectively)

Even though the thin occlusal veneers of the En Group have outperformed all the other veneers for both Vita enamic and E.max CAD (1328.5 N and $1231.2 \mathrm{~N}$ respectively), it was the low fracture resistance values of the ultrathin occlusal veneers (742.5N and 497.7N for Enamic and E.max CAD respectively) that decreased the overall mean values for the En Group.

The strategic enhancement in the fracture resistance values of thin occlusal veneers bonded to dentin when using IDS protocol as opposed to DDS protocol is significantly recognizable in this study and may be contributed to the fact that instantaneous direct sealing of the recently reduced dentin surface with bonding agent facilitates the focus on the treatment of different bonding substrates (enamel or dentin) one at a time independently owing to their different distinctive properties, therefore maximum bonding durability and strength could be achieved for both tooth tissues. ${ }^{(52-54)}$

The results of this study agree with the study done by Yazigi et al ${ }^{(9)}$ who assures the significant positive influence of the IDS usage. They declared that the freshly cut dentin provides the most promising bonding substrate only at the time of preparation and before contamination with any leftovers of the impression materials or temporary cements.

Additionally, higher bond strength values to dentin could be achieved by precuring of dentin bonding agents. In the DDS technique, the delayed unpolymerized adhesive layer that was left until the placement of the cement could result in collagen network or uncured dentin-resin hybrid layer collapse during restoration seating due to the pressure exerted at this stage. However, in the IDS technique, the resin is polymerized and has penetrated the hybrid layer. Besides, application of IDS allows the release of all the stresses and strains developed at the dentin bonding area before the placement of the restoration and the actual functional forces that will be applied on this tooth. ${ }^{55,56)}$

These results were coinciding with a lot of previous studies examining the fracture strength and bond strength values of different restorations upon using DDS and IDS protocols. They reported that the application of IDS protocol could result in biomimetic performance of thin adhesively luted occlusal veneers and tend to support the weak brittle ceramic restorations with subsequent increase in fracture resistance values and survival rates. ${ }^{(33,53)}$ Furthermore, it was assumed that application of IDS provides smooth surface and thus improve internal fit and adaptation of the restoration. ${ }^{(57)}$

Remarkably, it was stated that the weak thin occlusal veneers could act as strong thick occlusal veneers if the adhesive bonding mechanism is strong and durable. ${ }^{(32,58)}$ Nonetheless, it is worth mentioning that dentin exposure upon tooth preparation for occlusal veneers complicates the bonding capability. Self-etching primers provided better dentin bonding, however it has a negative effect on enamel surface. ${ }^{(59,60)}$ On the other hand, enamel surface performed better with total etch technique. Upon spotting this dilemma for the bonding capabilities of different tooth substrates and their nature, it comes up with the challenges that might be faced upon dealing with these clinical situations, especially upon choosing thin occlusal veneer restoration option. ${ }^{(8)}$

The results of this study showed statistically significant difference between Vita Enamic and 
E.max CAD where the former showed higher fracture resistance values upon bonding to enamel or DDS Dentin. Enamic occlusal veneers also yielded higher fracture resistance values than E.max CAD with IDS Dentin, however, the difference observed was statistically insignificant.

Each of the two materials has different properties and each one has an advantage that could improve the survival rates of occlusal veneers; The high fracture toughness and good bonding capabilities due to effective surface roughness using hydrofluoric acid etchant give lithium disilicate glass ceramic its unique nature. ${ }^{(29,30)}$ However, it seems that this material is very sensitive regarding the reduction of its occlusal thickness as the groups including IPS E.max CAD ultra-thin occlusal veneers with 0.3$0.6 \mathrm{~mm}$ thickness showed very low fracture strength values when bonded to enamel $(497.7 \mathrm{~N})$ or bonded to dentin with DDS protocol $(444.7 \mathrm{~N})$.

It is of significance to draw attention to the great improvement of lithium disilicate glass ceramics ultrathin occlusal veneers upon bonding to dentin only with IDS protocol which exceeds the average biting forces intraorally even for patients with bruxism.

On the other hand, Vita Enamic showed better results when used in minimum thickness $(0.3-0.6 \mathrm{~mm})$ regarding bonding to enamel $(742.5 \mathrm{~N})$ or bonding to dentin with DDS protocol $(727.4 \mathrm{~N})$. These results recommend its use in minimal thickness but only in normal bites without any parafunctional habits. This material seems not to be affected by reduced thicknesses as lithium disilicate. These results may be attributed to the greater flexibility that was given to the ceramic network of the hybrid ceramics after being infiltrated with polymers which lessens the crack propagation property that was recorded as a common problem with the ceramic materials. ${ }^{(61,62)}$

Many previous studies had explained the importance of the slight mismatch of the modulus of elasticity between the restoration and the supporting tissues or substrates ${ }^{(3,17,51,63)}$ and its positive effect on the fracture strength of these restorations. Restorative materials that better matches the modulus of elasticity of enamel or dentin gives better stress distribution and hence better fracture resistance values. ${ }^{(64)}$ In the current study, it appears that the slight mismatch between the elastic moduli (E) of the restorative material and bonding substrate has its impact when we are dealing with IPS E.max CAD. Better results were obtained when IPS E.max $\mathrm{CAD}(\mathrm{E}=95 \mathrm{GPa})$ was bonded to enamel $(\mathrm{E}=80 \mathrm{GPa})$ than when bonded to dentin with DDS protocol $(\mathrm{E}=19 \mathrm{GPa})$ -

On the other hand, although the elastic modulus of Vita Enamic ( $E=30 \mathrm{GPa})$ is much less than enamel, it still gives the best results when bonded to enamel than upon bonding to dentin with DDS protocol. This may be due to the assumption that the higher the elastic modulus of the bonding substrate, the greater the fracture resistance values as the forces needed to fracture the restoration are supposed to be greater. ${ }^{(65)}$

Upon comparing both materials used in this study bonded to dentin substrate with DDS, Vita Enamic gives better results than IPS E.max CAD. This confirms the importance of the slight mismatch between the elastic moduli of the restorative material and bonding substrate. On the other hand, the presence of enamel margins all around in all samples even in the dentin group might had resulted in better bond strength and stability.

The normal masticatory force in the maxillary premolar region ranges from $222 \mathrm{~N}$ to $422 \mathrm{~N}$ and ranges from $500-800 \mathrm{~N}$ in bruxism. ${ }^{(6,66)}$ Therefore, it was concluded that all the fracture resistance values of all samples were above the normal masticatory forces. However, the IPS e.max samples with 0.3$0.6 \mathrm{~mm}$ bonded to either enamel or dentin with DDS protocol were below the masticatory forces in bruxers.

The results of this study showed statistically significant difference between bonding protocols for 
both ceramics at thickness of 0.3-0.6mm (Ultra-thin occlusal veneers) where bonding of E.max CAD on either enamel or DDS dentin bonding substrates showed the worst results with mean values of $497.7 \mathrm{~N}$ and $444.7 \mathrm{~N}$ respectively. Upon increasing the thickness of the occlusal veneers to $0.5-0.8 \mathrm{~mm}$ (thin occlusal veneers), the fracture resistance values increase for all bonding substrates regarding both ceramic materials with remarkable significant increase for the enamel bonding substrate.

This was in harmony with the results of Sasse et al ${ }^{(7)}$ who revealed the worst results with 0.3$0.6 \mathrm{~mm}$ thickness of lithium disilicate glass ceramic occlusal veneers bonded to enamel substrate while the samples with $0.7-1 \mathrm{~mm}$ thickness performed favorably after cyclic loading and fracture resistance values irrespective of the type of the bonding substrate. On the other hand, the results of the research done by Krummel et al ${ }^{(8)}$ disagree with our results. They revealed average fracture resistance values with 1631-3391 $\mathrm{N}$ for the ultra-thin CAD/ CAM lithium disilicate glass ceramics with 0.3 $0.6 \mathrm{~mm}$ occlusal thickness. They concluded that their values were exceptionally higher than some similar studies ${ }^{(7,45)}$ because of using the selective enamel etching technique before application of self-etching primer which could guarantee strong bond strength to both enamel and dentin. They showed a statistical significance between groups involving both enamel and dentin upon comparing self-etch technique only or in conjunction with selective enamel etching step. Another study confirmed the same concept on comparing selective enamel etching technique to self-etching technique. They correlate the improved fracture resistance values of the samples bonded with selective etching technique to the stability of the adhesively bonded occlusal veneers in the enamel marginal area. ${ }^{(59)}$

Valenzuela et $\mathrm{al}^{\left({ }^{(3)}\right.}$ studied the effect of reduction of the occlusal veneer thickness on fracture resistance of glass ceramics. They obtained higher fracture strength values for occlusal veneers with $0.3 \mathrm{~mm}$ thickness than that with $0.6 \mathrm{~mm}$ thickness regardless of the bonding substrate and they linked their results to the successful adhesive bonding. These results were contradicting to the results of this study.

Extracted natural teeth were used in this study as a bonding substrate that gives a real clinically simulating condition to the bonding process. Although the teeth were meticulously chosen to decrease the variances in between the samples, the slight differences in the anatomy, dimensions, the time of extraction, the amount of enamel or dentin might have resulted in some variations among the results which is considered as a limitation. As a matter of fact, the prediction of the cause of failure in such multilayered ultrathin structures is hard as it may be related to many interchangeable factors as the actual strength of the material itself, the bonding substrate, the bonding mechanism, and the tooth type. Thus, further investigations are required to examine the fracture strength of thin and ultrathin occlusal veneers on molars rather than premolars with special attention to the different bonding mechanisms and minimal thickness of the restoration.

\section{CONCLUSIONS}

From the results of this study, it was concluded that:

1. Reduction of thickness of the occlusal veneers must be cautiously applied according to the suitable clinical conditions only.

2. Reduction of the lithium disilicate glass ceramics occlusal veneers to $0.3-0.6 \mathrm{~mm}$ negatively affects the fracture resistance values on bonding to either enamel or dentin with DDS protocol.

3. Ultrathin occlusal veneers $(0.3-0.6 \mathrm{~mm})$ is not ideal in any clinical situation when lithium disilicate glass ceramic is the chosen restorative 
material. However, this minimal thickness $(0.3 \mathrm{~mm}-0.6 \mathrm{~mm})$ could be a favorable option on using hybrid ceramics yet still could not be fully guaranteed in high-loading forces as patients with bruxism.

4. Alternatively, increasing the occlusal thickness to $0.5-0.8 \mathrm{~mm}$ could be promising upon bonding with either enamel only or dentin with enamel outer margin with both ceramic and hybrid ceramic materials.

5. Ultra-thin occlusal veneers with $0.3-0.6 \mathrm{~mm}$ thickness must be used carefully with both materials upon bonding to dentin (IDS must be applied).

\section{REFERENCES}

1. Ioannidis A, Muhlemann S, Ozcan M, Husler J, Hammerle C. Ultra-thin occlusal veneers bonded to enamel and made of ceramic or hybrid materials exhibit load-bearing capacities not different from conventional restorations. J Mech Behav of Biomed Mater 2019;90:433-440.

2. Lussi A, Schlueter N, Rakhmatullina E, Ganss C. Dental erosion-an overview with emphasis on chemical and histopathalogical aspects. Caries Res. 2011;45:2-12.

3. Valenzuela E, Sotero da Cunha P, Bittencourt H. Fracture load of CAD/CAM ultrathin occlusal veneers luted to enamel or dentin.J Esthet Restor Dent 2020;1-6.

4. Loomans B, Opdam N, Attin T, Bartlett D, Edelhoff D,Frankenberger R, et al. Severe tooth wear: Europeanconsensus statement on management guidelines. J AdhesDent 2017;19:111-9.

5. Heck K, Paterno H, Lederer A, Litzenburger F, Hickle R, Kunzelmann KH. Fatigue resistance of ultrathin CAD/ CAM ceramic and nanoceramic composite occlusal veneers. Dent Mater 2019;35:1370-1377.

6. Huang X, Zou L, Yao R, Wu S, Li Y. Effect of preparation design on the fracture behaviour of ceramic occlusal veneers in maxillary premolars. J Dent 2020;97:1-11.

7. Sasse M, Krummel A, Klosa K, Kern M. Influence of restoration thickness and dental bonding surface on the fracture resistance of full-coverage occlusal veneers made from lithium disilicate ceramic 2015;31:907-915.

8. Krummel A, Garling A, Sasse M, Kern M. Influence of bonding surface and bonding methods on the fracture re- sistance and survival rate of full-coverage occlusal veneers made from lithium disilicate ceramic after cyclic loading. Dent Mater 2019; 35:1351-1359.

9. Yazigi C, Kern M, Chaar MS. Influence of various bonding techniques on the fracture strength of thin CAD/CAM-fabricated occlusal glass-ceramic veneers. Journal of Mechanical Behavior of Biomedical Materials 2017; 75:504-511.

10. Ferrando-Cascales A, Astudillo-Rubio D, Pascual-Moscardo A, Delgado-Gaete A. A facially driven complete-mouth rehabilitation with ultrathin CAD-CAM composite resin veneers for a patient with severe tooth wear: A minimally invasive approach. J Prosthet Dent 2020;123:537-47.

11. Halim $\mathrm{CH}$. Fracture resistance of a newly proposed occlusal veneer design using two different CAD/CAM ceramic materials. EDJ 2018;64:2899-2915.

12. Gresnigt M, Cune M, de Roos J, Ozcan M. Effect of immediate and delayed dentin sealing on the fracture resistance, failure type and weilbull characteristics of lithiumdisilicate laminate veneers. Dent Mater 2016;32:e73-e81.

13. Mazurek K, Mierzwinska-Nastalska E, Molak R, Kozuchowski M, Pakiela Z. Strength and thickness of the layer of materials used for ceramic veneers bonding. Acta Bioengineering and Biomechanics 2012;14(3):75-78.

14. Al-Akhali M, Chaar MS, Elsayed A, Samran A, Kern M. Fracture resistance of ceramic and polymer-based occlusal veneer. Journal of Mechanical Behavior of Biomedical Materials 2017;74:245-250.

15. Moshaverinia A. Review of the modern dental ceramic restorative materials for esthetic dentistry in the minimally invasive age. Dent Clin N Am 2020;64:621-631.

16. Angerame D, De Biasi M, Agostinetto M, Franzo A, Marchesi G. Influence of preparation design on marginal adaptation and failure load of full-coverage occlusal veneers after thermomechanical aging simulation. J Esthet Restor Dent 2019;31:280-289.

17. Ma L, Guess PC, Zhang Y.Load-bearing properties of minimal-invasive monolithic lithium disilicate and zirconia occlusal onlays: finite element and theoretical analyses. Dent. Mater. 2013;29:742-751.

18. Egbert JS, Johnson AC, Tantbirojn D, Versluis A. Fracture strength of ultrathin occlusal veneer restorations made from CAD/CAM composite or hybrid ceramic materials. Oral. Sci. Int. 2015;12:53-58.

19. De Munck J, Van Meerbeek B, Yoshida Y, Inoue S, Vargas 
M, Suzuki K, Lambrechts P, Vanherle G. Four-year water degradation of total-etch adhesives bonded to dentin. J. Dent. Res.2003; 82:136-140.

20. Collares K, Corr^ea MB, Laske M, Kramer E, Reiss B, Moraes RR, Huysmans MCD, Opdam NJM. A practicebased research network on the survival of ceramic inlay/ onlay restorations. Dent. Mater.2016 32, 687-694.

21. Kuper NK, Opdam NJM, Bronkhorst EM, Huysmans, MCD. The influence of approximal restoration extension on the development of secondary caries. J. Dent. 2012; 40: 241-247.

22. Magne P, Douglas WH. Porcelain veneers: dentin bonding optimization and biomimetic recovery of the crown. Int $\mathrm{J}$ Prosthodont 1999;12:111-21.

23. Peumans M, De Munck J, Fieuws S, Lambrechts P, VanherleG, Van Meerbeek B. A prospective ten-year clinical trial of porcelain veneers. J Adhes Dent 2004;6:65-76.

24. Beier US, Kapferer I, Burtscher D, Dumfahrt H. Clinical performance of porcelain laminate veneers for up to 20 years. Int J Prosthodont 2012;25:79-85.

25. De Munck J, Van Landuyt K, Peumans M, Poitevin A, Lambrechts P, Braem M, Van Meerbeek B. A critical review of the durability of adhesion to tooth tissue: methods and results. J Dent Res 2005;84:118-32.

26. Kuijper M, Cune MS, Tromp Y, Gresnigt MM. Cyclic loading and load to failure of lithium disilicate endocrowns: Influence of the restoration extension in the pulp chamber and the enamel outline. Journal of Mechanical Behavior of Biomedical Materials.2020;105:1-7.

27. Gresnigt MM, Cune MS, Schuitemaker J, van der Made SAM, Meisberger EW, Magne P, Ozcan M. Performance of ceramic laminate veneers with immediate dentine sealing: an 11 year prospective clinical trial. Dent. Mater. 2019;35:1042-1052.

28. Van den Breemer CRG, Cune MS, Ozcan M, Naves LZ, Kerdijk W, Gresnigt MM. Randomized clinical trial on the survival of lithium disilicate posterior partial restorations bonded using immediate or delayed dentin sealing after 3 years of function. J. Dent.2019; https://doi.org/10.1016/j. jdent.2019.02.001.

29. Romanini-junior JC, Hirata R, Bonfante EA, Bordin D, Kumagai RY, Fardin VP, Coelho PG, Reis AF. Monolithic CAD/CAM laminate veneers: Reliability and failure modes. Dent. Mater.2020;36:724-732.
30. Saleh AM, Al-Ani M, AlRawi T, Al-Edressi G. An invitro comparison of fracture resistance of three $\mathrm{CAD} /$ CAM ceramic materials for fabricating veneer. Saudi Dental J 2020. Article in press. https://doi.org/10.1016/j. sdentj.2020.03.013.

31. Albelasy EH, Hamama HH, Tsoi JKH, Mahmoud SH. Fracture resistance of CAD/CAM occlusal veneers: A systematic review of laboratory studies. Journal of Mechanical Behavior of Biomedical Materials 2020;110:1-12.

32. Guess PC, Schultheis S, Wolkewitz M, Zhang Y, Strub JR.Influence of preparation design and ceramic thicknesses on fracture resistance and failure modes of premolar partialcoverage restorations. J Prosthet Dent 2013;110:264-73.

33. Hofsteenge JW, Hogeveen F, Cune MS, Gresnigt MM. Effect of immediate dentine sealing on the aging and fracture strength of lithium disilicate inlays and overlays. Journal of the Mechanical Behavior of Biomedical Materials 2020;110: 1-7.

34. Nakamura T, Tanaka H, Yatani H: In vitro study on marginal and internal fit of CAD/CAM all-ceramic crowns. Dent Mater J 2005; 24 : 456-9.

35. Lima JM, Souza AC, Anami LC, Bottino MA, Melo RM, Souza RO. Effects of thickness, processing technique, and cooling rate protocol on the flexural strength of a bilayer ceramic system. Dent. Mater. 2013; 29:1063-1072.

36. Rekow ED, Silva NR, Coelho PG, Zhang Y, Guess P, Thompson VP. Performance of dental ceramics: challenges for improvements. J. Dent. Res. 2011;90:937-952.

37. Ahlers MO, Morig G, Blunck U, Hajto J, Probster L, Frankenberger R. Guidelines for the preparation of CAD/CAM ceramic inlays and partial crowns. Int $\mathrm{J}$ Comput Dent. 2009; 12:309-325.

38. Kern M, Beuer F, Frankenberger R, Kohal R, Kunzelmann K, Mehl A, Pospiech P, Reiss B, Wiedhahn K. All-Ceramic-at a Glance. 6th ed. 2015. AG für Keramik in der Zahnheilkunde eV, Ettlingen.

39. Preis V, Dowerk T, Behr M, Kolbeck C, Rosentritt M. Influence of cusp inclination and curvature on the in vitro failure and fracture resistance of veneered zirconia crowns. Clin. Oral. Investig.2014;18:891-900.

40. Sornsuwan T, Ellakwa A, Swain MV. Occlusal geometrical considerations in all ceramic pre-molar crown failure testing. Dent Mater 2011;27:1127-1134.

41. Erickson R, Barkmeier WW, Kimmes NS. Bond strength 
of self-etch adhesives to pre-etched enamel. Dent Mater 2009; 25:1187-1194.

42. Sheets JL, Wilcox CW, Barkmeier WW, Nunn ME. The effect of phosphoric acid pre-etching and thermocycling on self-etching adhesive enamel bonding. J Prosthet Dent 2012;107:102-108.

43. Van Landuyt KL, Kanumilli P, De Munck J, Peumans M, Lambrechts P, Van Meerbeek B. Bond strength of a mild self-etch adhesive with and without prior acid-etching. J Dent 2006;34:77-85.

44. Fortin D, Swift Jr EJ, Denehy GE, Reinhardt JW. Bond strengthand microleakage of current dentin adhesives. Dent Mater 1994;10:253-8.

45. Clausen JO, Abou Tara M, Kern M, 2010. Dynamic fatigue and fracture resistance of non-retentive all-ceramic fullcoverage molar restorations. Influence of ceramic material and preparation design. Dent Mater 2010;26:533- 538.

46. Al-Akhali M, Kern M, Elsayed A, Samran A, Chaar MS. Influence of thermomechanical fatigue on the fracture strength of CAD-CAM-fabricated occlusal veneers. J Prosthet Dent 2019; 121: 644-650.

47. Morresi AL, D’Amario M, Capogreco M, Gatto R, Marzo G, D'Arcangelo C, Monaco A. Thermal cycling for restorative materials: Does a standardized protocol exist in laboratory testing? A literature review. J Mech Behav Biomed Mater. 2014 Jan;29:295-308.

48. Nawafleh N, Hatamleh M, Elshiyab S, Mack F. Lithium disilicate restorations fatigue testing parameters: A Systematic Review. J Prosthodont. 2016 Feb;25(2):116-26.

49. DeLong R, Douglas WH. Development of an artificial oral environment for the testing of dental restoratives: bi-axial force and movement control. J Dent Res. 1983;62:32-36.

50. Oyafuso DK, Ozcan M, Bottino MA, Itinoche MK. Influence of thermal and mechanical cycling on the flexural strength of ceramics with titanium or gold alloy frameworks. Dent Mater. 2008;24:351-356.

51. Emam ZN, Aleem NA. Influence of different materials and preparation designs on marginal adaptation and fracture resistance of CAD/CAM fabricated occlusal veneers. EDJ 2020;66:1-14.

52. Jayasooriya P, Pereira PN, Nikaido T, Tagami J. Efficacy of a resin coating on bond strengths of resin cement to dentin. J Esthet Restor Dent 2003;15:105-113.

53. Magne P, So WS, Cascione D. Immediate dentin sealing supports delayed restoration placement. J Prosthet Dent 2007;98:166-174.
54. Ozturk N, Aykent F. Dentin bond strengths of two ceramic inlay systems aftercementation with three different techniques and one bonding system. J Prosthet Dent 2003;89:275-281.

55. Park JW, Ferracane JL. Measuring the residual stress in dental composites using a ring slitting method. Dent Mater 2005; 21: 882-889.

56. Lee JI, Park SH. The effect of three variables on shear bond strength when luting a resin inlay to dentin. Oper Dent 2009;34:288-292.

57. Dietschi D, Monasevic M, Krejci I, Davidson C. Marginal and internal adaptation of class II restorations after immediate or delayed composite placement. J Dent 2002;30:259-69.

58. Bindl A, Luthy H, Mörmann WH. Strength and fracture pattern of monolithic CAD/CAM-generated posterior crowns. Dent Mater 2006;22:29-36.

59. Frankenberger R, Lohbauer U, Roggendorf MJ, Naumann M,Taschner M. Selective enamel etching reconsidered: betterthan etch-and-rinse and self-etch? J Adhes Dent 2008;10:339-44.

60. Hikita K, Van Meerbeek B, De Munck J, Ikeda T, Van LanduytK, Maida T, et al. Bonding effectiveness of adhesive lutingagents to enamel and dentin. Dent Mater 2007;23:71-80.

61. Ustun S, Ayaz EA. Effect of different cement systems and aging on the bond strength of chairside CAD-CAM ceramics.J Prosthet Dent 2021;125(2):334-339.

62. Leung B, Tsi J, Matinlinna J, Pow E. Comparison of mechanical properties of three machinable ceramics with an experimental fluorophlogopite glass ceramic. J Prosthet Dent 2015:114:440-446.

63. Sclichting LH, Maia HP, Baratieri LN, Magne P. Noveldesign ultra-thin CAD/CAM composite resin and ceramic occlusal veneers for the treatment of severe dental erosion. J Prosthet Dent 2011; 105:217-226.

64. Thompson VP, Rekow DE. Dental ceramics and the molar crown testing ground. J Appl Oral Sci 2004;12:26-36.

65. Abu-Izze FO, Ramos GF, Borges ALS, Anami LC, Bottino MA. Fatigue behavior of ultrafine tabletop ceramic restorations. Dent Mater 2018;34:1401-1409.

66. Sathyanarayana HP, Premkumar S. Assessment of maximum voluntary bite force in children and adults with normal occlusion. Int J Pharmac Scien Health care 2012;2(1): 64-70. 